\title{
High levels of sulphadoxine-pyrimethamine resistance Pfdhfr-Pfdhps quintuple mutations: a cross sectional survey of six regions in Tanzania
}

\author{
Sungwa I Matondo ${ }^{1}$, Godfrey S Temba ${ }^{1}$, Adelaida A Kavishe ${ }^{1,2}$, Julius S Kauki ${ }^{1}$, Akili Kalinga ${ }^{3}$,
} Marco van Zwetselaar ${ }^{1}$, Hugh Reyburn ${ }^{1,4}$ and Reginald A Kavishe ${ }^{1 *}$

\begin{abstract}
Background: In 2006, the first-line anti-malarial drug treatment in Tanzania was changed from sulphadoxine-pyrimethamine (SP) to artemether-lumefantrine (ALu), an artemisinin-based combination (ACT), since when the use of SP has been restricted for intermittent preventive treatment in pregnancy (IPTp). A number of Plasmodium falciparum mutations are known to be associated with resistance to SP, but it is not known if the prevalence of these mutations is increasing or decreasing under the conditions of reduced levels of SP use. This study reports on the current SP resistant quintuple Pfdhfr-Pfdhps mutations in six regions of Tanzania.

Methods: Finger-prick blood on filter paper and rapid diagnostic test strips from $P$. falciparum-positive individuals of all age groups attending health facilities in six regions of Tanzania between June 2010 and August 2011 were obtained. Using chelex-100 extracted DNA, genotyping was done for mutations on codons 51, 59 and 108 of Pfdhfr and 437 and 540 of Pfdhps genes using PCR-RFLP technique.

Results: A total of 802 malaria-positive samples were screened and genotyped. The prevalence of Pfdhfr 511 , Pfdhps $437 \mathrm{G}$ and 540E varied between the regions ( $p<0.001$ ) whereas Pfdhfr 59R (FE 10.79, $p=0.225$ ) and $108 \mathrm{~N}$ (FE 10.61, $p=0.239$ ) did not vary between the regions. The Pfdhfr triple mutant was above $84 \%$ and close to fixation levels in all regions, whereas the Pfdhps double mutation ranged from 43.8 to $97 \%$ between the regions. The quintuple mutant (IRNGE) was the most prevalent in all regions and it varied significantly from 37.5 to $90.2 \%\left(x^{2}=1.11, p<0.001\right)$.

Conclusions: There is evidence of persistent high levels of SP resistance markers in Tanzania with evidence of quintuple mutations that are likely to become fixed in the population. This threatens the future of SP not only in IPTp programmes, but as a combination drug for ACT. Continuous monitoring of SP-IPTp efficacy should be encouraged subsequent to searching for alternative drugs for IPTp in East Africa.
\end{abstract}

\section{Background}

Tanzania introduced sulphadoxine-pyrimethamne (SP) as first-line treatment drug for uncomplicated malaria in 2001, replacing chloroquine (CQ), which had been the first-line since the 1970s [1]. Before it was declared first-line, SP was already in use as second-line drug and resistance had already developed [2,3]. This led to a rapid spread of SP resistance and eventually SP was replaced with the current artemisinin-based combinational therapy (ACT) by the end of 2006 [4]. Due to

\footnotetext{
* Correspondence: rekavishe@yahoo.com

'Kilimanjaro Christian Medical University College and Kilimanjaro Clinical Research Institute, Moshi, Tanzania

Full list of author information is available at the end of the article
}

safety concerns for ACT use during pregnancy, especially in the first trimester, SP has continued to be used in intermittent preventive treatment of malaria in pregnancy (IPTp) and infants (IPTi). For IPTp, two or more doses of SP are administered after the first trimester at intervals of at least one month apart. The importance of SP-IPTp in prevention of malaria in pregnancy and the resulting outcomes, such as low birth weight, abortion, premature birth, perinatal death, and maternal mortality, have been documented globally and WHO has continued to recommend SP-IPTp use [5-8]. SP resistance has however continued to rise and several studies have reported reduced protection of SP-IPT programmes in areas where $\mathrm{SP}$ resistance is high [9-11]. 
SP resistance is caused by mutation on two genes, the dihydrofolate reductase (Pfdhfr) and the dihydropteroate synthetase (Pfdhps) genes. Three Pfdhfr mutations: N51I, C59R and S108N, referred to as the triple mutation, and the Pfdhps mutations: A437G and G540E, referred to as the double mutation, collectively form the quintuple mutations [12,13]. An additional mutation on Pfdhps 581 has been associated with high level of SP resistance and a strong predictor of SP-IPTp failure [14] and in addition to the quintuple forms the sextuple mutation. In East Africa SP resistance has reached over $90 \%$ and in some places the prevalence of the quintuple mutation is approaching fixation levels [15]. In Tanzania only two studies in Igombe-Mwanza and Korogwe-Tanga have documented the prevalence of quintuple mutation in 2008/2011 period. All other studies have used samples collected before or during the transition from SP to $\mathrm{ACT}$ in 2006. It is thus not clear whether SP resistance is decreasing or increasing in the advent of its restricted use. The current study set out to investigate the current SP resistance based on quintuple mutations in Tanzania.

\section{Methods}

Samples collected through collaboration with ongoing studies in six regions of mainland Tanzania between June 2010 and August 2011 were used in this study. In Coastal Region the sample involved pregnant women attending the Kibiti health centre for intermittent preventive treatment of malaria. Sampling from all other regions involved all age groups. Finger-prick blood on filter paper (Whatman-3) or rapid diagnostic test kits (Mwanza samples) from febrile patients attending various health facilities in the respective regions were collected after patients' or children's guardians had consented to the use of their blood samples for malarial genetic studies. The study sites included Mwanza (Misungwi district) and Kagera (Muleba district) around Lake Victoria in the north-western zone, Tanga (Bondo village) in the northeastern zone, Mtwara (Tandahimba and Mtwara-Urban) and Coastal Region (Kibiti-Rufiji) in the south-eastern zone, and Mbeya (Kyela and Rungwe districts) in the south-western zone. The malaria-positive rapid diagnostic test (RDT) strips or dried filter-paper blood spots were stored in desiccant at room temperature. Malaria parasite DNA was extracted using chelex-100 method as described previously [16]. Genotyping for Pfdhps and Pfdhfr was performed using PCR-RFLP methods described by others $[17,18]$. In short, nested PCR were performed followed by restriction digestion of the secondary products. For Pfdhfr Tsp509I, XmnI and AluI were used for positions 51, 59 and 108 respectively whereas for Pfdhps 437 and 540 AvaII and FokI were used, respectively. For each enzyme there were digestion control sites as previously described [17] in addition positive controls were used in each experiment. Digestion products were eluted on $2 \%$ agarose gel (Invitrogen, USA) stained with ethidium bromide and visualized under UV light. All PCR reagents and restriction endonucleases were purchased from $\mathrm{New}$ England Biolabs (Ipswich, MA, USA). Primers were purchased from Biolegio (Nijmegen, the Netherlands). Prevalence was calculated as the percentage of wild type or mutants out of the new total samples genotyped. Very few mixed infections were observed in this study and were excluded from the analysis as it was not possible to include them in haplotype analysis. The study received ethical approval from the Kilimanjaro Christian Medical University College Ethical Board subsequent to the National Institute for Medical Research Ethics approval obtained in the collaborating projects.

\section{Results}

A total of 802 P. falciparum positive blood samples were screened and genotyped; 785, 787, 765, 762 and 752 were successfully genotyped for mutations at codons 51 , 59, 108, 437 and 540 respectively; 707 (88\%) of the 802 were successfully analyzed for the quintuple haplotypes. At codons 51, 59, 108 and 437, 0.6, 1.4, 1.3 and 1.4\% of the genotyped samples had mixed genotypes. No mixed genotypes were observed at codon 540. Since the percentages were low, samples with mixed genotypes were excluded from haplotype calculation. Significant differences in prevalence of Pfdhfr 51I (FE 10.79, p < 0.001), Pfdhps 437G $\left(\chi^{2}=1.5, \mathrm{p}<0.001\right)$ and 540E $\left(\chi^{2}=1.12\right.$, $\mathrm{p}<0.001)$ were observed between the regions. However, the prevalence of Pfdhfr 59R and $108 \mathrm{~N}$ mutations was not different between the regions (FE 10.79, $\mathrm{p}=0.225$ and FE 10.61, $\mathrm{p}=0.239$, respectively).

Pfdhfr mutations were the most prevalent (Figure 1) with the triple mutant (IRN) ranging from 84.4 (Coastal) to $96.6 \%$ (Tanga) compared to Pfdhps double mutant (GE) which ranged from 43.8 to $97 \%$ (Table 1 ). Both the triple mutant and the double mutants were statistically different but when Coastal region was excluded the distribution of the IRN triple mutant was no longer different (FE 2.75, $\mathrm{p}=0.594)$. The wild type Pfdhfr (NCS) and Pfdhps (AK) were detected at very low levels $(0.1 \%$ and $5.1 \%$ respectively) (Table 1 ).

Six common quintuple haplotypes were observed from the analysis (Table 2) with overall prevalence ranging from 1.8 to $76.9 \%$ depicted in Figure 2. An additional 13 minor haplotypes with prevalence less than $1 \%$ were grouped as "others" and constituted only $4.1 \%$ of the overall haplotypes. These include NRNGK (0.6\%), IRSAK (0.4\%), NCNGE (0.4\%), NCNAK(0.3\%), NCNGK (0.3\%), NRNAE (0.1\%), IRSAE (0.1\%), IRSGK (0.1\%), ICNGE (1.1\%), NRNAK (0.1\%), ICNGK (0.1\%), NCSGE (0.1\%) and ICNAE (0.1\%). The IRNGE haplotype (quintuple mutant) was the most prevalent haplotype in all regions and it varied 

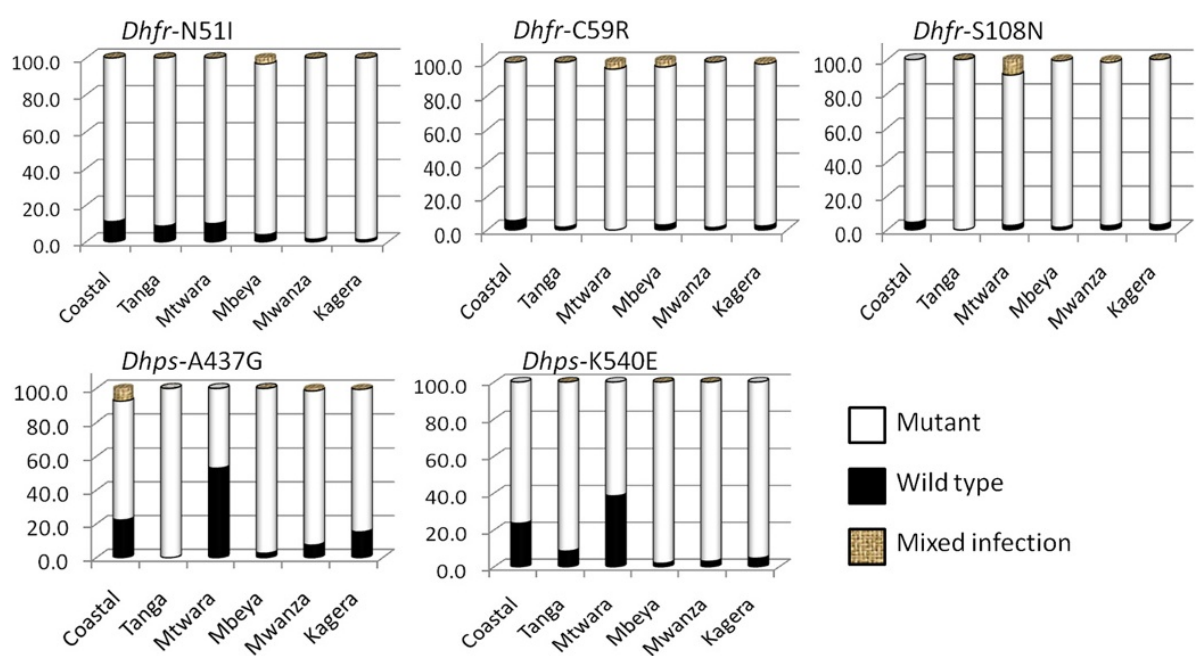

Figure 1 Prevalence of Pfdhfr and Pfdhps mutations in Tanzania. X-axis represents the six regions sampled and y-axis presents percentage prevalence calculated as total number of mutants or wild types per total number of samples per region.

significantly across the regions $\left(\chi^{2}=1.11, \mathrm{p}<0.001\right)$ (Table 2). Tanga, Mbeya, Mwanza and Kagera regions had the highest prevalence of the quintuple mutation compared to Coastal and Mtwara regions (Table 2 and Figure 2).

\section{Discussion}

Selection for SP resistance markers in Tanzania has remained high even after the replacement of SP for firstline treatment of uncomplicated malaria in 2006. The selection for individual Pfdhfr and Pfdhps mutations is very high throughout Tanzania. Comparing individual mutations, Pfdhfr 59R is already fixed in Mtwara region while $108 \mathrm{~N}$ and Pfdhps 437 are fixed in Tanga (Bondo). In Korogwe-Tanga, the 51I, 59R and $108 \mathrm{~N}$ were already above 95\% in 2006 [14] and in Mbeya-Matema, in 2005 the 51I, 59R, $108 \mathrm{~N}, 437 \mathrm{G}$, and 540E were 93, 80, 97.7, 78.6 and $77.4 \%$, respectively [19]. A similar increase was observed in Mwanza Region. Between 2010 and 2011 the prevalence of 51I, 59R, $108 \mathrm{~N}, 437 \mathrm{G}$, and 540E in IgombeMwanza was 75, 82.5, 94.8, 74, and 69.5\%, respectively which is comparable to the current findings [20].
The wild type Pfdhfr haplotype NCS was reported at $1.9 \%$ in Tanga-Korogwe in the period 2008/2010 [21] but in this study it was not detected, it was detected in Mwanza at $0.8 \%$. This indicates disappearance of the wild type haplotypes as the mutants increase.

Furthermore, compared to studies conducted between 2006 and 2007 around the time when SP was withdrawn as first line drug, the triple mutant (IRN) was $90-96.4 \%$ in Tanga (Korogwe), 74\% in Coastal (Rufiji) and Mtwara/ Lindi regions while in Mbeya (Matema) it was $82.6 \%$ in 2005 [19,22-24], thus there has been a continuous selection for the Pfdhfr triple mutants to date. Similarly, from around 2006 the double mutant (GE) and the quintuple respectively have continued to increase from 63 and $75 \%$ in Tanga [14,22], and 81 and 64\% in Mbeya [19] while the GE increased from 57\% in Lindi/Mtwara. There was no statistical difference in the distribution of the IRN across regions indicating homogeneity in SP selection pressure throughout the country.

The Pfdhps double (GE) mutant varied between the regions. While the prevalence was lower in Mtwara

Table 1 Prevalence of Pfdhfr triple and Pfdhps double mutants in Tanzania

\begin{tabular}{|c|c|c|c|c|c|c|c|c|c|c|c|c|}
\hline \multirow[b]{2}{*}{ Regions } & \multicolumn{7}{|c|}{ Pfdhfr n (\%) } & \multicolumn{5}{|c|}{ Pfdhps n (\%) } \\
\hline & IRN & IRS & ICN & NRN & $\mathrm{NCN}$ & NCS & Total & GE & GK & $\mathrm{AE}$ & AK & Total \\
\hline Coastal & $81(84.4)$ & $5(5.2)$ & $0(0)$ & $3(3.1)$ & $7(7.3)$ & $0(0)$ & 96 & $59(61.5)$ & $13(13.5)$ & 15 (15.6) & $9(9.4)$ & 96 \\
\hline Tanga & 112 (96.6) & $0(0)$ & $2(1.7)$ & $2(1.7)$ & $0(0)$ & $0(0)$ & 116 & 107 (92.2) & $9(7.8)$ & $0(0)$ & $0(0)$ & 116 \\
\hline Mtwara & $59(92.2)$ & $2(3.1)$ & $0(0)$ & $3(4.7)$ & $0(0)$ & $0(0)$ & 64 & $28(43.8)$ & $8(12.5)$ & $12(18.8)$ & $16(25.0)$ & 64 \\
\hline Mbeya & $127(96.2)$ & $3(2.3)$ & $2(1.5)$ & $0(0)$ & $0(0)$ & $0(0)$ & 132 & $128(97.0)$ & $1(0.8)$ & $3(2.3)$ & $0(0)$ & 132 \\
\hline Mwanza & $126(96.2$ & $2(1.5)$ & $2(1.5)$ & $0(0)$ & $0(0)$ & $1(0.8)$ & 131 & $122(93.1)$ & $0(0)$ & $5(3.8)$ & $4(3.1)$ & 131 \\
\hline Kagera & $158(94.0)$ & $6(3.6)$ & $4(2.4)$ & $0(0)$ & $0(0)$ & $0(0)$ & 168 & $148(88.1)$ & $1(0.6)$ & $12(7.1)$ & $7(4.2)$ & 168 \\
\hline Total & $663(93.8)$ & $18(2.5)$ & $10(1.4)$ & $8(1.1)$ & $7(1.0)$ & $1(0.1)$ & 707 (100) & $592(83.7)$ & $32(4.5$ & $47(6.6)$ & $36(5.1)$ & 707 (100) \\
\hline
\end{tabular}


Table 2 Prevalence of Pfdhfr-Pfdhps common haplotypes in six regions of Tanzania

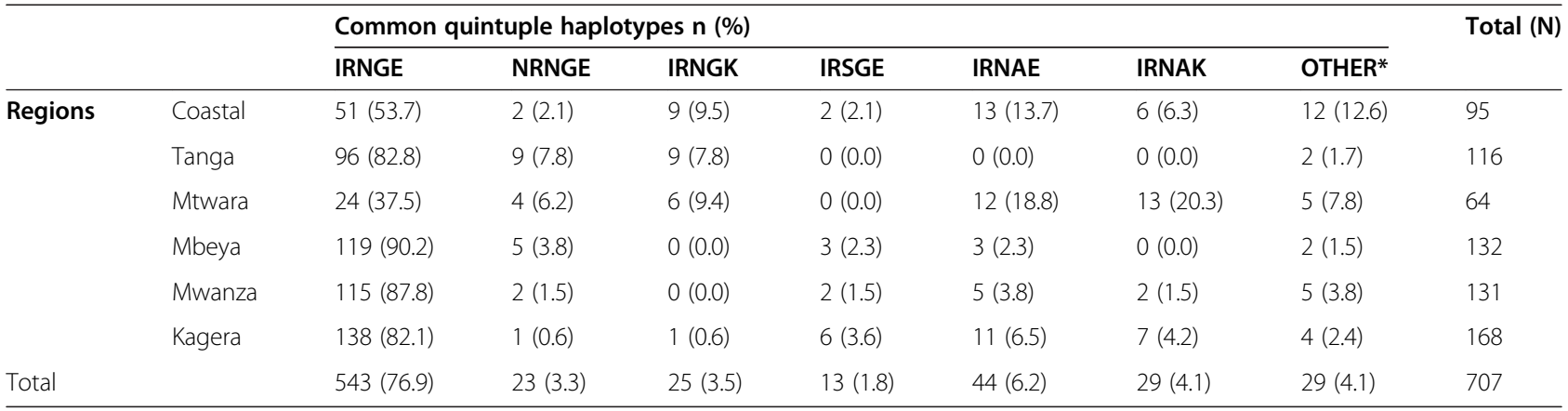

*Other haplotypes include: NRNGK, IRSAK, NCNGE, NCNAK, NCNGK, NRNAE, IRSAE, IRSGK, ICNGE, NRNAK, ICNGK, NCSGE and ICNAE.

and Coastal regions, highest levels were observed in Mbeya, Mwanza, Tanga and Kagera. This may be accounted for by inter regional variations in the use of SP especially during or before SP became first line treatment drug. Before 2001 SP was second line drug and CQ was the first line. During this time SP resistance had already occurred. This contributed to a rapid spread of resistance after SP was made first line in 2001. In 2005 Mbeya registered exceptionally high levels of GE (81\%) [19] and in the current study Mbeya is the leading with highest levels of SP resistance (Tables 1 and 2, Figure 1).

Six common quintuple haplotypes were observed. The observed high levels of the quintuple mutation in all regions derive from the high levels observed with the triple and double mutations of Pfdhfr and Pfdhps. 7The low levels of double mutant (GE) in Coastal and Mtwara regions resulted into low levels of the quintuple

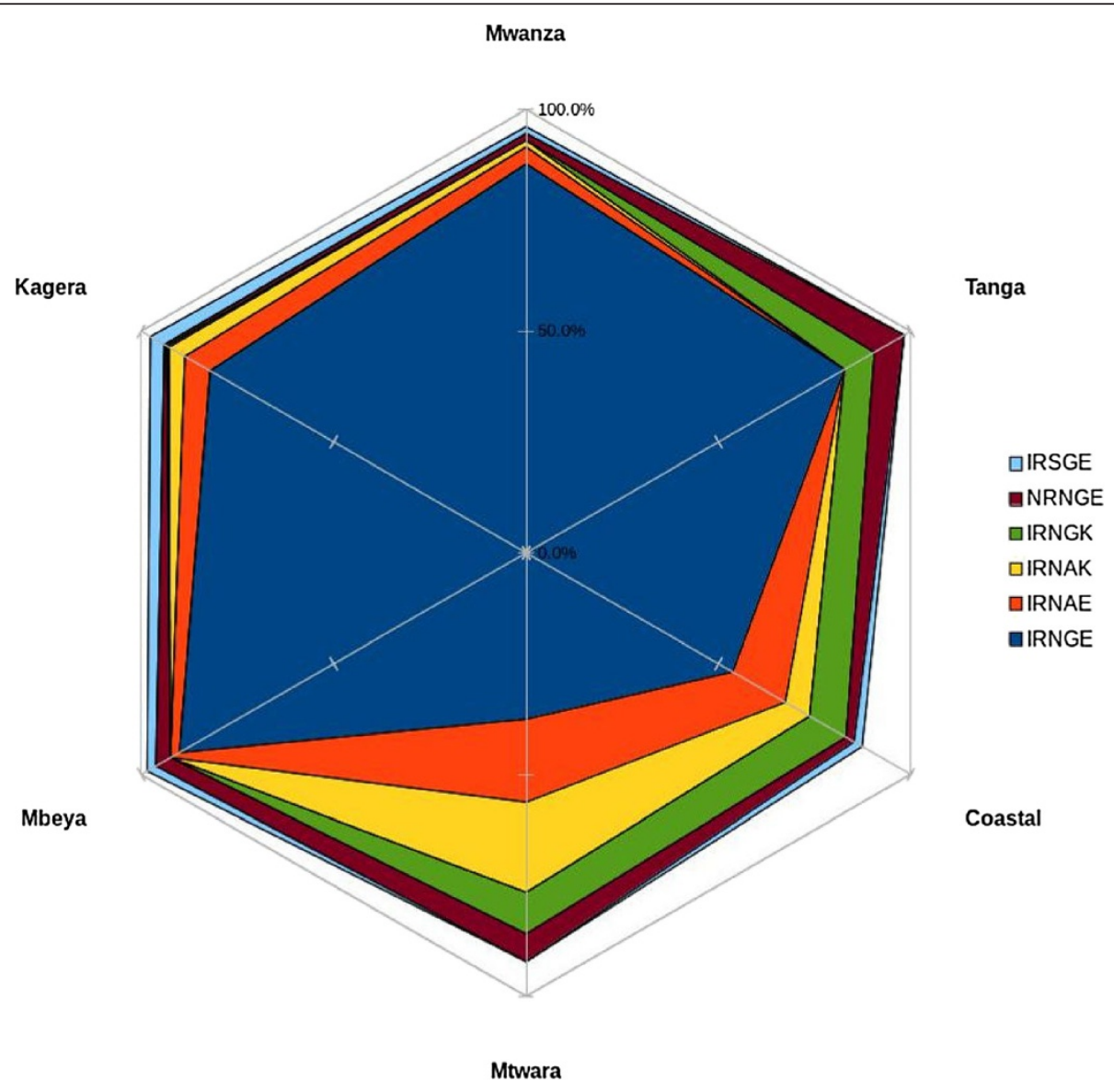

Figure 2 Prevalence of Pfdhfr-dhps common quintuple haplotypes in Tanzania. 
mutation in these regions. These findings are comparable to recent studies in other East African countries. In western Kenya samples obtained from pregnant women between 2008 and 2009 were found to harbour more than 90\% Pfdhps double mutant and more than $80 \%$ quintuple mutation [25]. In Mozambique SP resistance quintuple mutation was reported to be above $75 \%$ in 2008 while the triple mutation had reached $100 \%$ (fixation) [26]. These reports point to high SP resistance in the East African region as opposed to the West African region where SP resistance based on the quintuple mutation is still low in most countries, thus SP-IPT is still effective [27-29].

The prevalence of the quintuple mutation in the parasite confers high level SP resistance. In East Africa high levels of this haplotype are likely to compromise the importance of SP-IPTp [30]. Several studies have shown that although implementation of SP-IPTp does not prevent malaria infection during pregnancy, especially in the presence of high prevalence of SP-resistance markers $[14,31,32]$, there is a significant protection against severe outcomes of pregnancy in malaria, such as low birth weight, maternal and neonatal mortality, especially when more than two doses of IPTp are administered [33]. This led to WHO's continued recommendation for SP-IPTp at any level of quintuple mutations [34]. However, continued SP-IPTp is likely to exacerbate the spread of the highly resistant Pfdhps mutation 581 previously reported to associate with IPTp failure in East Africa $[14,25]$. Thus, aside from the WHO recommended $>$ two doses of SP-IPTp, the high prevalence of SP resistance markers observed in Tanzania and elsewhere in East Africa calls for careful and continuous evaluation of SP-IPTp efficacy and on the usefulness of SP in artemisinin combinations. There is a need to screen pregnant mothers for malaria parasites even when they are already on IPTp in order to identify early treatment failure of the intervention [35]. Recent studies show that CQ withdrawal from use for a number of years has reversed resistance based on prevalence of Pfcrt resistance marker [36,37]. This was possible since CQ use was totally banned making its availability to both health facilities and local drug vendors difficult. A survey done in 2007 documented CQ use in Tanzania at $0.5 \%$ and in Malawi at $0.8 \%$ [38]. This led to the reported recovery of CQ susceptibility in Tanzania and Malawi. Conversely, due to continued use of SP for IPTp, SP is readily available in both public and the private sector making its restriction to only IPTp impossible. In the current situation it is unlikely that selfmedication with SP can be prevented especially due to its low cost compared to ACT, which may also explain the observed high prevalence of SP resistance markers despite its replacement with ACT. Use of SP-artesunate combination is also another selection factor for SPresistance markers, however, in Tanzania SP-AS is not used instead artemether-lumefantrine (ALu) is the approved ACT. Furthermore, it is expected as the quintuple mutation continues to rise towards fixation, the Pfdhps $581 \mathrm{G}$ mutation considered to confer SP superresistance when in combination with the $540 \mathrm{E}$ will continue to rise. It is important for the responsible authorities to consider restricting SP to IPTp only, through restricting its general prescription and its availability to local drug vendors. An alternative drug for IPTp is urgently needed.

\section{Conclusion}

In this study prevalence of SP resistance based on quintuple mutations in Tanzania is high, approaching fixation levels. This trend has been observed in other parts of East Africa. The spread of SP super-resistance is expected with continued SP use and may lead to poor SP-IPTp outcome despite continued recommendation by the WHO. An urgent search for alternative drugs for IPTp in East Africa is required.

\section{Competing interests}

The authors have declared that they have no competing interests.

\section{Authors' contributions}

SIM participated in study design, performed the experiments, interpreted the data and drafted the manuscript. GST participated in performing the experiments and revised the manuscript. AAK and AK supervised sample collection in the field and revised the manuscript. JSK and MvS participated in data analysis and reviewed the manuscript. HR participated in study design and reviewed the manuscript. RAK conceived the idea, designed the study, analysed the data and wrote the manuscript. All authors read and approved the final version of the manuscript.

\section{Acknowledgements}

RAK was supported by a postdoctoral fellowship grant under the Training Health Researchers into Vocational Excellence in East Africa (THRiVE) consortium funded by the Wellcome Trust Grant Number 087540.

\section{Author details}

${ }^{1}$ Kilimanjaro Christian Medical University College and Kilimanjaro Clinical Research Institute, Moshi, Tanzania. ${ }^{2}$ Kilimanjaro Christian Medical Centre, Moshi, Tanzania. ${ }^{3}$ National Institute for Medical Research, Tukuyu Centre, Tanzania. ${ }^{4}$ London School of Hygiene and Tropical Medicine, London, UK.

Received: 17 December 2013 Accepted: 13 April 2014

Published: 21 April 2014

\section{References}

1. Taverne J: Tanzania phases out chloroquine for the treatment of malaria. Trends Parasitol 2001, 17:360.

2. Eriksen J, Mwankusye S, Mduma S, Kitua A, Swedberg G, Tomson G, Gustafsson LL, Warsame M: Patterns of resistance and DHFR/DHPS genotypes of Plasmodium falciparum in rural Tanzania prior to the adoption of sulfadoxine-pyrimethamine as first-line treatment. Trans $R$ Soc Trop Med Hyg 2004, 98:347-353.

3. Gorissen E, Ashruf G, Lamboo M, Bennebroek J, Gikunda S, Mbaruku G, Kager PA: In vivo efficacy study of amodiaquine and sulfadoxine/ pyrimethamine in Kibwezi, Kenya and Kigoma, Tanzania. Trop Med Int Health 2000, 5:459-463.

4. Njau JD, Goodman CA, Kachur SP, Mulligan J, Munkondya JS, McHomvu N, Abdulla S, Bloland P, Mills A: The costs of introducing artemisinin-based 
combination therapy: evidence from district-wide implementation in rural Tanzania. Malar J 2008, 7:4.

5. Menendez C, Bardaji A, Sigauque B, Sanz S, Aponte JJ, Mabunda S, Alonso PL: Malaria prevention with IPTp during pregnancy reduces neonatal mortality. Plos One 2010, 5:e9438.

6. Verhoeff FH, Brabin BJ, Chimsuku L, Kazembe P, Russell WB, Broadhead $\mathrm{RL}$ : An evaluation of the effects of intermittent sulfadoxinepyrimethamine treatment in pregnancy on parasite clearance and risk of low birthweight in rural Malawi. Ann Trop Med Parasitol 1998, 92:141-150.

7. Verhoeff FH, Brabin BJ, Chimsuku L, Kazembe P, Broadhead RL: Malaria in pregnancy and its consequences for the infant in rural Malawi. Ann Trop Med Parasitol 1999, 93(Suppl 1):S25-S33.

8. WHO-MPAC: Malaria Policy Advisory Committee to the WHO conclusions and recommendations of September 2012 meeting. Malar J 2012, 11:424.

9. Gosling RD, Gesase S, Mosha JF, Carneiro I, Hashim R, Lemnge M, Mosha FW, Greenwood B, Chandramohan D: Protective efficacy and safety of three antimalarial regimens for intermittent preventive treatment for malaria in infants: a randomised, double-blind, placebo-controlled trial. Lancet 2009, 374:1521-1532.

10. Griffin JT, Cairns M, Ghani AC, Roper C, Schellenberg D, Carneiro I, Newman RD, Grobusch MP, Greenwood B, Chandramohan D, Gosling RD: Protective efficacy of intermittent preventive treatment of malaria in infants (IPTi) using sulfadoxine-pyrimethamine and parasite resistance. PLoS One 2010, 5:e12618.

11. Harrington WE, Mutabingwa TK, Kabyemela E, Fried M, Duffy PE: Intermittent treatment to prevent pregnancy malaria does not confer benefit in an area of widespread drug resistance. Clin Infect Dis 2011, 53:224-230.

12. Triglia T, Wang P, Sims PF, Hyde JE, Cowman AF: Allelic exchange at the endogenous genomic locus in Plasmodium falciparum proves the role of dihydropteroate synthase in sulfadoxine-resistant malaria. EMBO J 1998, 17:3807-3815

13. Wang P, Read M, Sims PF, Hyde JE: Sulfadoxine resistance in the human malaria parasite Plasmodium falciparum is determined by mutations in dihydropteroate synthetase and an additional factor associated with folate utilization. Mol Microbiol 1997, 23:979-986.

14. Gesase S, Gosling RD, Hashim R, Ord R, Naidoo I, Madebe R, Mosha JF, Joho A, Mandia V, Mrema H, Mapunda E, Savael Z, Lemnge M, Mosha FW Greenwood B, Roper C, Chandramohan D: High resistance of Plasmodium falciparum to sulphadoxine/pyrimethamine in northern Tanzania and the emergence of dhps resistance mutation at Codon 581. PLoS One 2009, 4:e4569.

15. Raman J, Mauff K, Muianga P, Mussa A, Maharaj R, Barnes Kl: Five years of antimalarial resistance marker surveillance in Gaza Province, Mozambique, following artemisinin-based combination therapy roll out PLoS One 2011, 6:e25992.

16. Polski JM, Kimzey S, Percival RW, Grosso LE: Rapid and effective processing of blood specimens for diagnostic PCR using filter paper and Chelex-100. Mol Pathol 1998, 51:215-217.

17. Jelinek T, Ronn AM, Curtis J, Duraisingh MT, Lemnge MM, Mhina J, Bygbjerg IC, Warhurst DC: High prevalence of mutations in the dihydrofolate reductase gene of Plasmodium falciparum in isolates from Tanzania without evidence of an association to clinical sulfadoxine/ pyrimethamine resistance. Trop Med Int Health 1997, 2:1075-1079.

18. University of Mayland School of Medicine: PCR-allele-specific restriction analysis (ASRA): protocols for Plasmodium falciparum drug resistance mutation analyses. University of Mayland; 2002. Accessed:10-5-2013.

19. Schonfeld M, Barreto MI, Schunk M, Maduhu I, Maboko L, Hoelscher M, Berens-Riha N, Kitua A, Loscher T: Molecular surveillance of drug-resistance associated mutations of Plasmodium falciparum in south-west Tanzania. Malar J 2007, 6:2

20. Kamugisha E, Jing S, Minde M, Kataraihya J, Kongola G, Kironde F, Swedberg G: Efficacy of artemether-lumefantrine in treatment of malaria among under-fives and prevalence of drug resistance markers in Igombe-Mwanza, north-western Tanzania. Malar J 2012, 11:58

21. Minja DT, Schmiegelow C, Mmbando B, Bostrom S, Oesterholt M, Magistrado P, Pehrson C, John D, Salanti A, Luty AJ, Lemnge M, Theander T, Lusingu J, Alifrangis M: Plasmodium falciparum mutant haplotype infection during pregnancy associated with reduced birthweight, Tanzania. Emerg Infect Dis 2013, 19: doi: 10.3201.

22. Alifrangis $M$, Lusingu JP, Mmbando B, Dalgaard MB, Vestergaard LS, Ishengoma D, Khalil IF, Theander TG, Lemnge MM, Bygbjerg IC: Five-year surveillance of molecular markers of Plasmodium falciparum antimalarial drug resistance in Korogwe District, Tanzania: accumulation of the $581 \mathrm{G}$ mutation in the $P$. falciparum dihydropteroate synthase gene. Am J Trop Med Hyg 2009, 80:523-527.

23. Malisa AL, Pearce RJ, Mutayoba BM, Abdullah S, Mshinda H, Kachur PS, Bloland $P$, Roper $C$ : The evolution of pyrimethamine resistant $\mathrm{dhfr}$ in Plasmodium falciparum of south-eastern Tanzania: comparing selection under SP alone vs SP + artesunate combination. Malar J 2011, 10:317.

24. Pearce RJ, Ord R, Kaur H, Lupala C, Schellenberg J, Shirima K, Manzi F, Alonso P, Tanner M, Mshinda H, Roper C, Schellenberg D: A communityrandomized evaluation of the effect of intermittent preventive treatment in infants on antimalarial drug resistance in southern Tanzania. J Infect Dis 2013, 207:848-859.

25. Iriemenam NC, Shah M, Gatei W, van Eijk AM, Ayisi J, Kariuki S, Vanden EJ, Owino SO, Lal AA, Omosun YO, Otieno K, Desai M, ter Kuile FO, Nahlen B, Moore J, Hamel MJ, Ouma P, Slutsker L, Shi YP: Temporal trends of sulphadoxine-pyrimethamine (SP) drug-resistance molecular markers in Plasmodium falciparum parasites from pregnant women in western Kenya. Malar J 2012, 11:134.

26. Raman J, Little F, Roper C, Kleinschmidt I, Cassam Y, Maharaj R, Barnes Kl: Five years of large-scale dhfr and dhps mutation surveillance following the phased implementation of artesunate plus sulfadoxine-pyrimethamine in Maputo Province, Southern Mozambique. Am J Trop Med Hyg 2010, 82:788-794.

27. Doumbo S, Ongoiba OA, Doumtabe D, Dara A, Ouologuem TD, Kayentao K, Djimde A, Traore B, Doumbo OK: [Prevalence of Plasmodium falciparum, anemia and molecular markers of chloroquine and sulfadoxinepyrimethamine resistance in delivered women in Fana, Mali] (in French). Bull Soc Pathol Exot 2013, 106:188-192.

28. Duah NO, Quashie NB, Abuaku BK, Sebeny PJ, Kronmann KC, Koram KA Surveillance of molecular markers of Plasmodium falciparum resistance to sulphadoxine-pyrimethamine 5 years after the change of malaria treatment policy in Ghana. Am J Trop Med Hyg 2012, 87:996-1003

29. Ndiaye M, Tine R, Faye B, Ndiaye JL, Diouf I, Lo AC, Sylla K, Dieng Y, Hallett $R$, Alifrangis M, Gaye O: Selection of antimalarial drug resistance after intermittent preventive treatment of infants and children (IPTi/c) in Senegal. C R Biol 2013, 336:295-300.

30. Allen EN, Little F, Camba T, Cassam Y, Raman J, Boulle A, Barnes KI: Efficacy of sulphadoxine-pyrimethamine with or without artesunate for the treatment of uncomplicated Plasmodium falciparum malaria in southern Mozambique: a randomized controlled trial. Malar J 2009, $8: 141$

31. Gutman J, Mwandama D, Wiegand RE, Ali D, Mathanga DP, Skarbinski J: Effectiveness of intermittent preventive treatment with sulfadoxinepyrimethamine during pregnancy on maternal and birth outcomes in Machinga district, Malawi. J Infect Dis 2013, 208:907-916.

32. Taylor SM, Antonia AL, Chaluluka E, Mwapasa V, Feng G, Molyneux ME, ter Kuile FO, Meshnick SR, Rogerson SJ: Antenatal receipt of sulfadoxinepyrimethamine does not exacerbate pregnancy-associated malaria despite the expansion of drug-resistant Plasmodium falciparum: clinical outcomes from the QuEERPAM study. Clin Infect Dis 2012, 55:42-50

33. Eisele TP, Larsen DA, Anglewicz PA, Keating J, Yukich J, Bennett A, Hutchinson P, Steketee RW: Malaria prevention in pregnancy, birthweight, and neonatal mortality: a meta-analysis of 32 national cross-sectional datasets in Africa. Lancet Infect Dis 2012, 12:942-949.

34. WHO-MPAC: Malaria Policy Advisory Committee to the WHO: conclusions and recommendations of March 2013 meeting. Malar J 2013, 12:213.

35. Lin JT, Mbewe B, Taylor SM, Luntamo M, Meshnick SR, Ashorn P: Increased prevalence of dhfr and dhps mutants at delivery in Malawian pregnant women receiving intermittent preventive treatment for malaria. Trop Med Int Health 2013, 18:175-178.

36. Laufer MK, Thesing PC, Eddington ND, Masonga R, Dzinjalamala FK, Takala SL, Taylor TE, Plowe CV: Return of chloroquine antimalarial efficacy in Malawi. N Engl J Med 2006, 355:1959-1966. 
37. Mohammed A, Ndaro A, Kalinga A, Manjurano A, Mosha JF, Mosha DF, van ZM, Koenderink JB, Mosha FW, Alifrangis M, Reyburn H, Roper C, Kavishe RA: Trends in chloroquine resistance marker, Pfcrt-K76T mutation ten years after chloroquine withdrawal in Tanzania. Malar J 2013, 12:415.

38. Frosch $A E$, Venkatesan M, Laufer MK: Patterns of chloroquine use and resistance in sub-Saharan Africa: a systematic review of household survey and molecular data. Malar J 2011, 10:116.

doi:10.1186/1475-2875-13-152

Cite this article as: Matondo et al:: High levels of sulphadoxine-

pyrimethamine resistance Pfdhfr-Pfdhps quintuple mutations: a cross

sectional survey of six regions in Tanzania. Malaria Journal 2014 13:152.

\section{Submit your next manuscript to BioMed Central and take full advantage of:}

- Convenient online submission

- Thorough peer review

- No space constraints or color figure charges

- Immediate publication on acceptance

- Inclusion in PubMed, CAS, Scopus and Google Scholar

- Research which is freely available for redistribution 